\title{
Idiopathic Interstitial Pneumonia
}

National Cancer Institute

\section{Source}

National Cancer Institute. Idiopathic Interstitial Pneumonia. NCI Thesaurus. Code C35714.

Non-infectious inflammation of the interstitial lung tissue. This category includes the desquamative interstitial pneumonia, usual interstitial pneumonia, lymphocytic interstitial pneumonia, acute interstitial pneumonia, and nonspecific interstitial pneumonia. 